\title{
Threshold voltage instability in SiC MOSFETs as a consequence of current conduction in their body diode
}

\author{
Oriol Avino-Salvado ${ }^{\mathrm{a}}$, Hervé Morel ${ }^{\mathrm{a}}$, Cyril Buttay ${ }^{\mathrm{a}, *}$, Denis Labrousse ${ }^{\mathrm{b}}$, Stéphane Lefebvre $^{\mathrm{b}}$ \\ ${ }^{a}$ Univ Lyon, INSA-Lyon, CNRS, Université Claude Bernard Lyon 1, École Centrale de Lyon, Laboratoire Ampère, F-69621, VIlleurbanne, France \\ ${ }^{b}$ CNRS, SATIE UMR 8029, F-94235 Cachan, France
}

\begin{abstract}
An antiparallel PiN diode is present in the structure of the SiC Power MOSFET. For simplicity, increased module current ratings, and cost reasons, it sometimes may be preferable to use this diode rather than to add an external Schottky diode to the MOSFET. However, $\mathrm{SiC}$ PiN diodes are sensitive to defects in the SiC crystal, and their performance can degrade rapidly.

In this paper, we assess the effect of several stresses on the characteristics of a SiC MOSFET, focusing on the stresses which involve the body diode. We demonstrate that using the body diode does not degrade its performances. However, it may produce a noticeable shift in the threshold voltage of the MOSFET. While this behaviour is observed for MOSFETs from one manufacturer, it is not exhibited for others, which indicates that it may be a consequence of some particular design or manufacturing parameters.
\end{abstract}

\section{Introduction}

$\mathrm{SiC}$ power devices are now commercially available from many manufacturers [1]. They offer dramatic improvements in performance over the ubiquitous Si power devices [2], especially regarding high voltage or high temperature [3]. SiC technology is progressing rapidly, and although devices still suffer from some reliability issues, they continuously improve.

Many issues regarding manufacturing yield or device reliability can be traced back to defects in the SiC crystal structure, such as screw dislocations [4] or micropipes [5]: when they do not alter a device's performance so much that it becomes useless, defects can cause a gradual drift in its performance. An example is the increase in forward voltage of PiN diodes as a result of the propagation of defects such as stacking faults [6]. Similar observations were made on $\mathrm{SiC}$ bipolar transistors [7]. These defects also cause increased leakage current, and therefore strongly affect high voltage devices [8]. SiC wafers with reduced defect density are now available, with less than 1000 dislocations $/ \mathrm{cm}^{2}$ over a $150 \mathrm{~mm}$ wafer [1], so crystal quality is no longer considered as a major issue which could impede the commercial success of $\mathrm{SiC}$.

Another set of issues affects SiC MOSFETs. It is related to the electron mobility in the channel [6] and the reliability of the oxide layer which forms the MOS structure [9]. The consequences are a higher on-state resistance (in the case of poor channel mobility) and a gradual drift in the threshold voltage of the MOSFET. In some extreme cases, such drift could cause the device to become normally-on [10]. As with the crystal defects, recent improvements in the technology have largely mitigated these issues, and SiC MOSFETs have de facto become

\footnotetext{
${ }^{*}$ Corresponding author

Email address: cyril.buttay@insa-lyon.fr (Cyril Buttay)
}

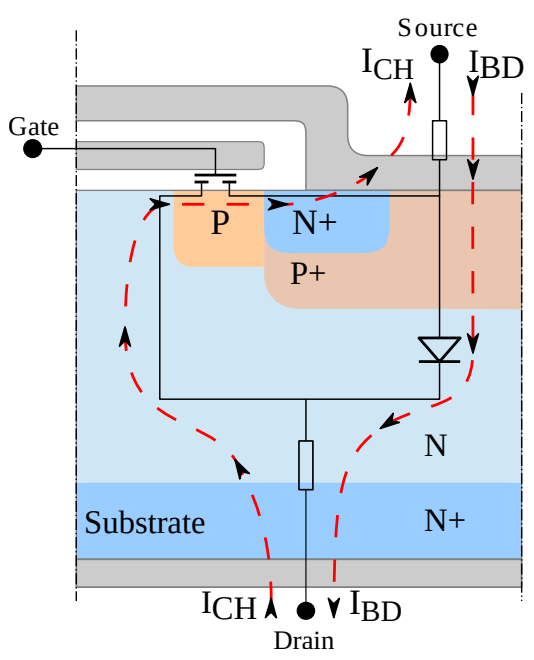

Figure 1: Cross section of a power MOSFET, showing the $\mathrm{I}_{B D}$ (body diode) and $\mathrm{I}_{C H}$ (channel) current paths.

the major SiC controlled switch. This is illustrated by the discontinuation of alternative devices such as Infineon's JFET of Fairchild's BJT.

With the improvement in technology, the $\mathrm{SiC}$ devices have now reached a point where they can be used in real applications, and the reliability studies have shifted from simple test setups to more complex configurations. An example is the body diode of the MOSFET. In inverter topologies, an antiparallel diode is usually connected to each transistor so current can flow during the "dead-time" periods (when both transistors of a half-bridge are in the off-state). Classically, a SiC Schottky (or Merged PiN-Schottky) diode is used, because it has a low forward voltage $\left(V_{F} \approx 1 \mathrm{~V}\right)$ and because it does not suffer from material degradation, as presented above. However, this requires two 
components per switch (a MOSFET and a diode). As the power MOSFET structure already contains a PiN diode (Fig. 1), a possibility is to use it instead of an external diode. This saves space (one die per switch instead of two) and money, at the cost of increased losses: PiN diodes have a higher forward voltage drop ( $V_{F} \approx 3 \mathrm{~V}$ instead of $1 \mathrm{~V}$ for Schottky diodes) and recovery charge (although not nearly as much as comparable Si diodes). However, as the dead-time period is short, usually a few percents of the switching period, the effect remains small. It is worth noting that a SiC MOSFET with an integrated Schottky diode was introduced in [11], but in most cases, the internal (body) diode has a PiN junction as described in Fig. 1.

A recent study [12] investigated the degradation of the PiN junction of a SiC MOSFET in realistic use cases (a 3-phase inverter). Only a very small change in forward voltage drop was observed (increase as well as decrease, indicating that stacking faults were not the predominant degradation mechanism at work). Overall, it was found that the body diode could be used as a functional element, in place of external diodes.

Here, we present the results of a similar investigation, where we not only monitored the changes in the forward voltage drop of body diodes $\left(V_{F}\right)$ but also in the threshold voltage of the corresponding MOSFET $\left(V_{t h}\right)$. As in [12], we observe no significant change in $V_{F}$ due to current flowing in the body diode. However, we observe a strong shift in $V_{t h}$ in at least one manufacturer's parts, which has not been reported so far.

The next section of this article presents the test protocols based on static (i.e. no switching) or dynamic (more realistic case) operations. The corresponding results are presented and discussed in section 3 .

\section{Test Protocol}

Except when explicitly mentioned, all tests presented here were performed on C2M0080120D SiC MOSFETs (Wolfspeed), which are rated at $1200 \mathrm{~V}$ and $80 \mathrm{~m} \Omega$. The devices were purchased in two batches (2014 and 2016): the first batch was used for the HTRB/CMB tests (see below), while the second was used for all the other tests (Q1, Q3, Q4 and HTGB). For the sake of simplicity, these devices are referred to as "Wolfspeed" MOSFETs in the text. All characterizations were performed using a Keysight B1505A curve tracer, at room temperature, $1 \mathrm{~h}$ after the end of the corresponding test (to allow enough time for the devices to cool down, but soon enough to minimize relaxation effects).

\subsection{Static Tests}

Five static test circuits are used: the first two are the "High Temperature Reverse Bias" (HTRB) and "High Temperature Gate Bias (HTGB) (Fig. 2, top), described in a JEDEC standard [13], which are classically used to evaluate the effects of a continuous bias on power devices. For the HTRB, a negative voltage source (here $-8 \mathrm{~V}$, corresponding to a value close to the absolute minimum voltage, $-10 \mathrm{~V}$, allowed in its datasheet [14]) is connected to the gate and source of the Device Under Test (DUT), and a positive voltage is applied between drain and

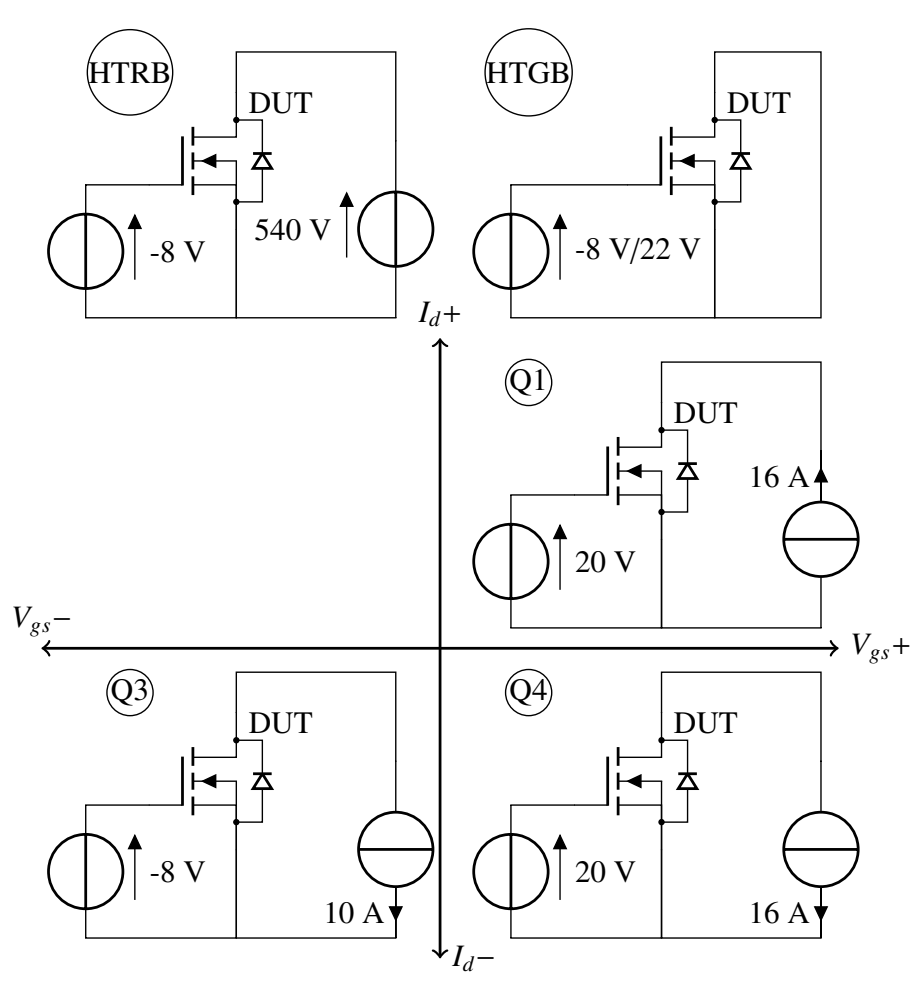

Figure 2: The five static test circuits: voltage bias on the drain (HTRB), negative or positive gate bias on the gate, with drain and source shorted (HTGB), and three tests with current bias (Q1 to Q4).

Table 1: Summary of the static tests conditions

\begin{tabular}{l|c|c|c|c|c|c}
\hline Test & $\begin{array}{c}\text { samples } \\
(\mathrm{N})\end{array}$ & $\begin{array}{c}V_{g s} \\
(\mathrm{~V})\end{array}$ & $\begin{array}{c}I_{d s} \\
(\mathrm{~A})\end{array}$ & $\begin{array}{c}V_{d s} \\
(\mathrm{~V})\end{array}$ & $\begin{array}{c}T_{j} \\
\left({ }^{\circ} \mathrm{C}\right)\end{array}$ & $\begin{array}{c}\text { Power } \\
(\mathrm{W})\end{array}$ \\
\hline \hline HTRB & 10 & -8 & 0 & 540 & 150 & 0 \\
\hline HTGB & 1 & -8 & 0 & 0 & 150 & 0 \\
\hline HTGB & 3 & 22 & 0 & 0 & 150 & 0 \\
\hline $1^{\text {st }}$ quadrant & 3 & 20 & 16 & 0 & 150 & 37.3 \\
\hline $3^{\text {rd }}$ quadrant & 3 & -8 & -10 & 0 & 150 & 37.3 \\
\hline $3^{\text {rd }}$ quadrant & 3 & -5 & -10 & 0 & 150 & 37.3 \\
\hline $4^{\text {th }}$ quadrant & 3 & 20 & -16 & 0 & 150 & 37.3 \\
\hline
\end{tabular}

source (here $540 \mathrm{~V}$, which corresponds to the bus voltage of the aeronautic HVDC standard [15]). For the HTGB, the drain and source terminals are shorted, and two gate levels were investigated here: $-8 \mathrm{~V}$ or $22 \mathrm{~V}$ (a value very close to the absolute maximum allowed). In these tests, no current flows through the DUT.

The three remaining test circuits are also depicted in Fig. 2 (configurations Q1, Q3 and Q4). They represent the three configurations which result in current flowing through the drain of the MOSFET: either the transistor is in the on-state $\left(V_{g s}=20 \mathrm{~V}\right.$, the recommended value in the datasheet) so the current flows through its channel $\left(I_{C H}\right.$ path in Fig. 1) in both directions (Q1 or $\mathrm{Q} 4)$, or the transistor is in the off-state $\left(V_{g s}=-8 \mathrm{~V}\right)$, so the current flows through its body diode $\left(I_{B D}\right.$ in Fig. 1). This latter configuration corresponds to Q3 in Fig. 2.

All tests are run at the maximum junction temperature allowed by the manufacturer $\left(150^{\circ} \mathrm{C}\right)$, to accelerate any degrada- 


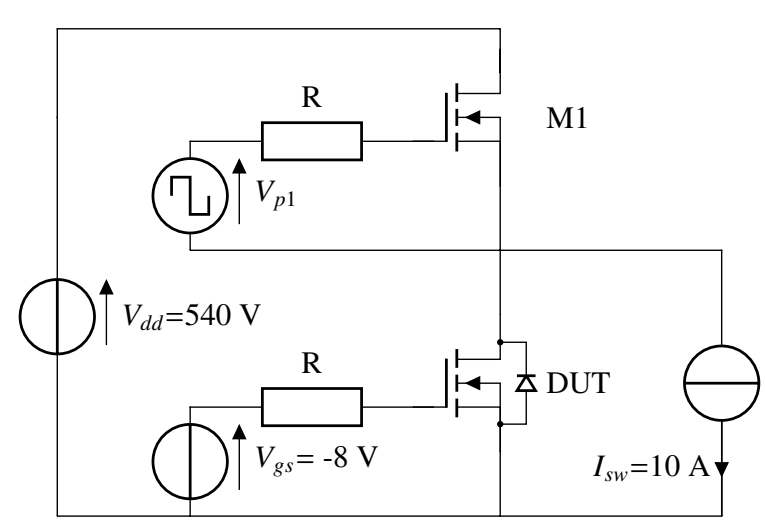

Figure 3: Circuit diagram of the CMB test.

tion mechanism which may occur. For the HTRB test, where no power is dissipated, this simply requires the DUT to be attached to a hot plate at a set temperature of $150{ }^{\circ} \mathrm{C}$. On the contrary, for the other tests from Fig. 2 (Q1, Q3 and Q4), the DUTs dissipate a large amount of power, because of their large drain current, resulting in a noticeable self-heating. To ensure that they still operate at $T_{J}=150^{\circ} \mathrm{C}$, they are mounted on temperature-regulated plates. Their thermal resistance "junction-to-plate" $\left(R_{T h_{J P}}\right)$ is then characterized using the drain current of the DUT in the saturation region $\left(V_{g s}=6 \mathrm{~V}, V_{d s}=8 \mathrm{~V}\right)$, both to generate selfheating of the device, and as a Temperature-Sensitive Electrical Parameter (TSEP, [16]) to monitor the corresponding temperature rise. $R_{T h_{J P}}$ is found to be $2.19 \mathrm{~K} / \mathrm{W}$. During the static tests, the drain current is set to $10 \mathrm{~A}$ when it flows through the body diode (quadrant Q3) and $16 \mathrm{~A}$ when it flows through the channel of the MOSFET (quadrants Q1 and Q4). Both conditions correspond to a power dissipation $\mathrm{P}=37 \mathrm{~W}$ when the DUT operates at $T_{J}=150{ }^{\circ} \mathrm{C}$. As a consequence, the temperature-regulated plates are set to a temperature $T_{P}$ of:

$$
T_{P}=T_{J}-P \times R_{T h_{J P}}=68.3^{\circ} \mathrm{C}
$$

A summary of the test conditions is given in Tab. 1. Tests in the Q3 quadrant were run for two values of $V_{g s}(-5 \mathrm{~V}$ and $-8 \mathrm{~V})$ as this parameter was found to have a strong effect on the results.

\subsection{Dynamic Tests}

The circuit diagram used for the dynamic tests is presented in Fig. 3. As can be seen in the theoretical waveforms presented in Fig. 4, the DUT experiences both high voltage when M1 is on, and high current (through its body diode, as the DUT remains off) when M1 is off. This operating mode is referred to as "Chopper-Mode Bias" (CMB).

An arbitrary switching frequency of $20 \mathrm{kHz}$ was chosen for the results presented here, with a $50 \%$ duty cycle ratio between M1 and the DUT. As for the static tests, the devices are attached to a temperature-regulated plate, set at the temperature required to achieve $T_{J}=150^{\circ} \mathrm{C}$. The temperature of the plate is calculated using eq. (1), where $\mathrm{P}$ is the power dissipated in the DUT when M1 is off.

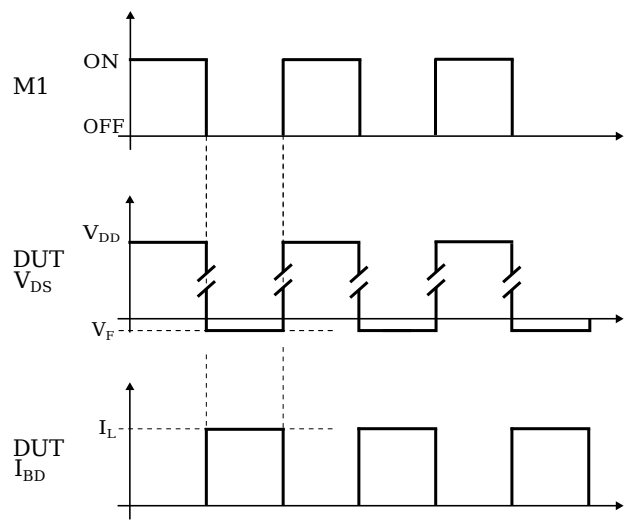

Figure 4: Principle of the CMB test bench: When M1 is in the off-stage, the current flows through the body diode of the DUT. When it is on, the full voltage $(540 \mathrm{~V})$ is applied between drain and source of the DUT.

\section{Results and Discussion}

\subsection{Static tests}

Fig. 5 presents the measurements of $V_{F}$ for quadrants 1 , 3 and 4. No significant evolution is observed over $100 \mathrm{~h}$ : the changes in $V_{F}$ during the tests are comparable to the experimental scattering, except for Q1, which show a slightly clearer trend, with a $2 \%$ drop in $V_{F}$ after $15 \mathrm{~h}$. For the HTRB and HTGB tests (not presented here because of space limitations), $V_{F}$ exhibits no significant change. As for $V_{F}$, the evolution of $R_{d s_{o n}}$ was also measured, but it is not presented here as this parameter shows no significant change, for any of the test conditions (HTRB, HTGB, Q1, Q3 or Q4).

The evolution of $V_{t h}$ is plotted in Figs. 7 and 6. It remains essentially constant for the HTRB, HTGB, Q1 and Q4 tests. For Q3, however, $V_{t h}$ shows a dramatic drop over time $(-40 \%$ in $60 \mathrm{~h}$ ) when the MOSFETs are driven with $V_{g s}=-8 \mathrm{~V}$. The tests were repeated on 3 other devices, with $V_{g s}=-5 \mathrm{~V}$, a value which still ensures proper blocking of the transistor (initial $V_{t h}$ values were found to be $1.12-1.45 \mathrm{~V}$ ). The drop in $V_{t h}$ then appears to slow down considerably (orange line in Fig. 7).

Q3 corresponds to the condition in which the biasing current flows through the body diode of the MOSFET (Fig. 2); in Q1 and Q4 the current flows through the channel, while HTRB and HTGB correspond to a voltage-only bias. However, contrary to what was expected, no degradation of the body diode is observed (increase in $V_{F}$ caused by the propagation of stacking faults). Instead, the devices exhibit a large drop in $V_{t h}$, for the Q3 test condition. Such decrease is undesirable, as it makes the device harder to block, and makes it more vulnerable to electromagnetic interference (which might cause spurious turn-ons).

\subsection{Dynamic Tests}

Using the CMB setup (Fig. 3), devices from three manufacturers (Wolfspeed, ST Microelectronics and Rohm Semiconductors) were tested. The results are given in Fig. 8 for $V_{t h}\left(V_{F}\right.$ and $R_{d s_{o n}}$ were also monitored, but as with the static tests, they show no significant evolution). For Wolfspeed devices, a large $V_{t h}$ drop is observed (Fig. 8). On the contrary, almost no change is observed for ST's or Rohm's MOSFETs. 


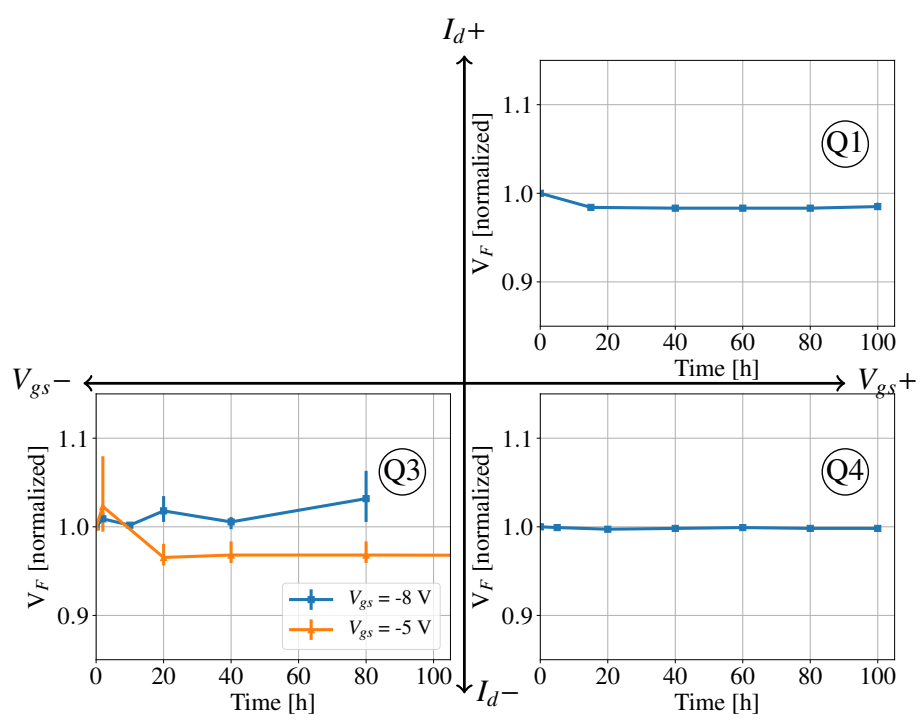

Figure 5: Evolution of the forward voltage of the MOSFETs (normalized for $\mathrm{t}=0$, measured at $I_{d}=10 \mathrm{~A}, V_{g s}=-8 \mathrm{~V}, T_{J}=25^{\circ} \mathrm{C}$ ) during the current-bias tests described in Fig. 2 (3 samples per test for Q1, Q3 and Q4, 10 samples for HTRB). All tests were run at $T_{J}=150{ }^{\circ} \mathrm{C}$.

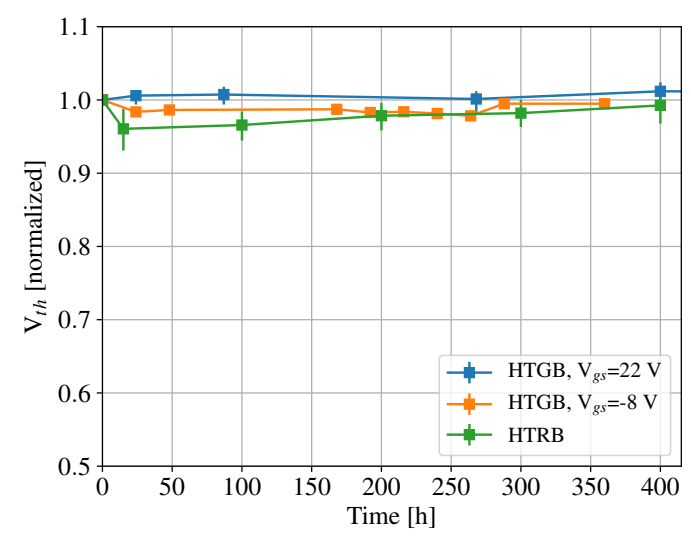

Figure 6: Evolution of the threshold voltage for HTRB (10 samples) and HTGB conditions ( 1 sample for $V_{g s}=-8 \mathrm{~V}, 3$ samples for $G_{g s}=22 \mathrm{~V}$ ). All tests were run at $T_{J}=150^{\circ} \mathrm{C}$.

A comparison between the $V_{t h}$ drop observed using the CMB test setup and the static Q3 test setup is presented in Fig. 9. Note that the $\mathrm{x}$-axis corresponds to the time during which current actually flows through the body diode (i.e. $100 \%$ of the test time for $\mathrm{Q} 3$, and $50 \%$ of the test time for $\mathrm{CMB}$ ). Tests were performed using two values of $V_{g s}:-5$ and $-8 \mathrm{~V}$. In both cases, a good agreement is found: most of the $V_{t h}$ drop can be associated with the use of the body diode of the DUT.

In an attempt to identify the cause behind the differences in behaviour among components from different manufacturers, additional investigations were conducted. Fig. 10 presents the results of HTGB tests performed on devices identical to those used for Fig. 8. Contrary to the data presented in section 3.1, these tests were run at $T_{J}=200^{\circ} \mathrm{C}$, to strongly accelerate ageing. It should be noted that while this temperature is within the allowed range for ST's MOSFETs [17], it exceeds Rohm's $\left(175^{\circ} \mathrm{C},[18]\right)$ or Wolfspeed's $\left(150^{\circ} \mathrm{C},[14]\right)$. The high tem-

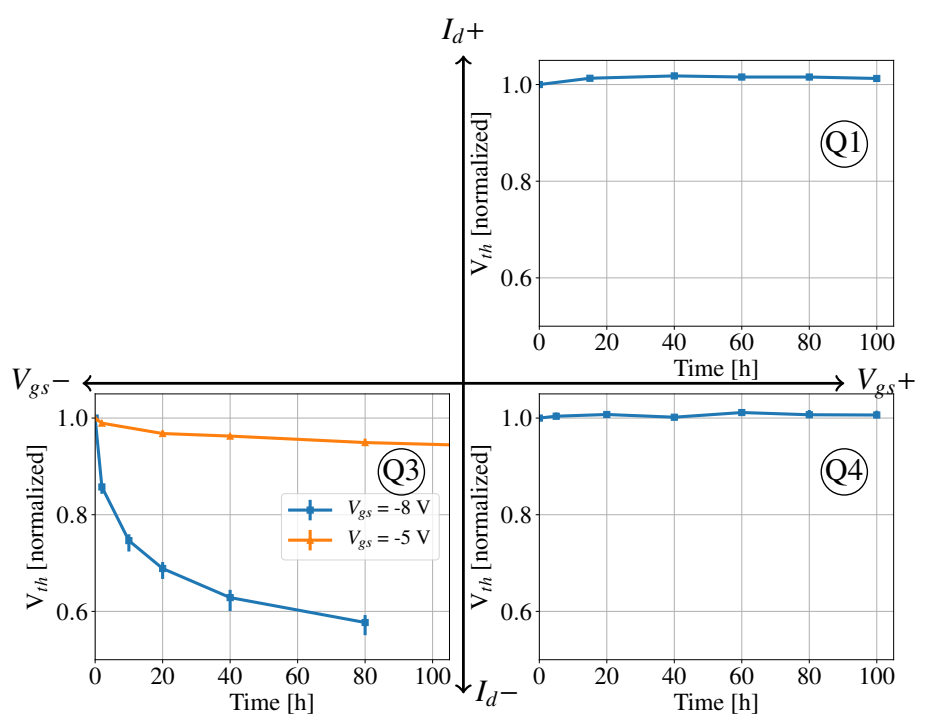

Figure 7: Evolution of the threshold voltage of the MOSFETs (normalized for $\mathrm{t}=0$, measured at $I_{d}=100 \mu \mathrm{A}, V_{d d}=1 \mathrm{~V}, T_{J}=25^{\circ} \mathrm{C}$ ) during the static tests described in Fig. 2 ( 3 samples per test). All tests were run at $T_{J}=150^{\circ} \mathrm{C}$.

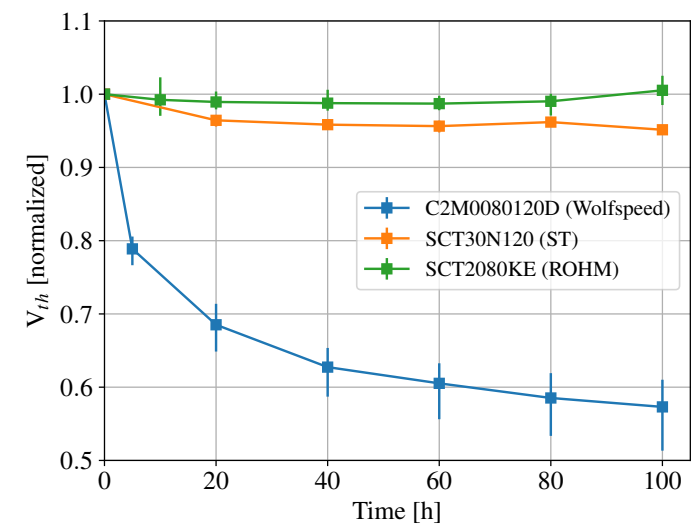

Figure 8: Evolution of the $V_{t h}$ of MOSFETs from three different manufacturers, as a function of time in conditions of CMB ( 3 samples per test), data normalized for $\mathrm{t}=0$, measured at $I_{d}=100 \mu \mathrm{A}, V_{d d}=1 \mathrm{~V}, T_{J}=25^{\circ} \mathrm{C}$ and $V_{g s}=-8 \mathrm{~V}$. All tests were run at $T_{J}=150^{\circ} \mathrm{C}$.

perature was nonetheless required to observe any difference in behaviour: as can be seen in Fig. 10, while all devices exhibit a small shift in $V_{t h}(\approx 6 \%)$ within $50 \mathrm{~h}$ of test, MOSFETs from $\mathrm{ST}$ and Rohm tend to stabilize over time at around $\mathrm{a} \approx 10 \%$ increase in $V_{t h}$. On the contrary, Wolfspeed MOSFETs exhibit a continuous increase in $V_{t h}$, with $30 \%$ more over $1000 \mathrm{~h}$. Only positive-bias HTGB tests were performed at $200{ }^{\circ} \mathrm{C}$, because of limited sample availability.

Additionally, cross sections were performed on devices from the three manufacturers. They were prepared mechanically (cut with a diamond saw, and polished using diamond particles down to $1 \mu \mathrm{m}$, followed by $50 \mathrm{~nm}$ oxide suspension), and observed using a scanning electron microscope (SEM, Vega 3, Tescan). It is found that all three devices exhibit a planar gate structure, with some differences in the size of their features. The resolution of the images is limited, and very fine details such as oxide thickness cannot be observed clearly. There is, 


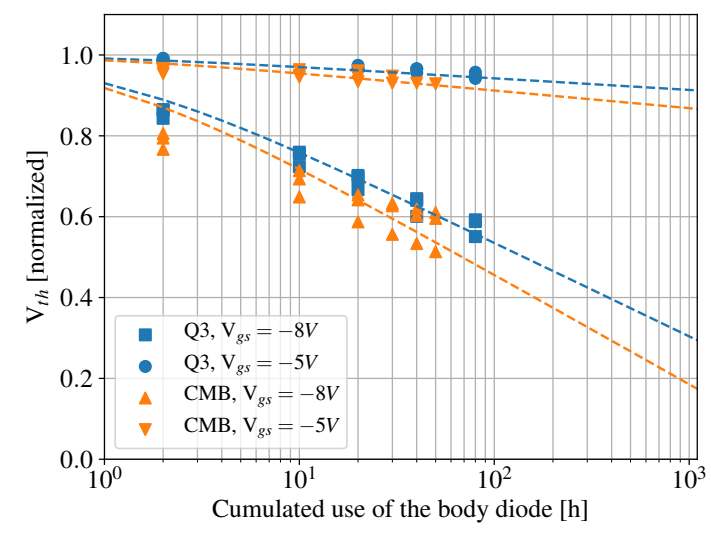

Figure 9: Evolution of the $V_{t h}$ as a function of time for the Static ( $3^{\text {rd }}$ quadrant) and $\mathrm{CMB}$ tests ( 3 samples per test), data normalized for $\mathrm{t}=0$, measured at $I_{d}=100 \mu \mathrm{A}, V_{d d}=1 \mathrm{~V}, T_{J}=25^{\circ} \mathrm{C}$. All tests were run at $T_{J}=150^{\circ} \mathrm{C}$.

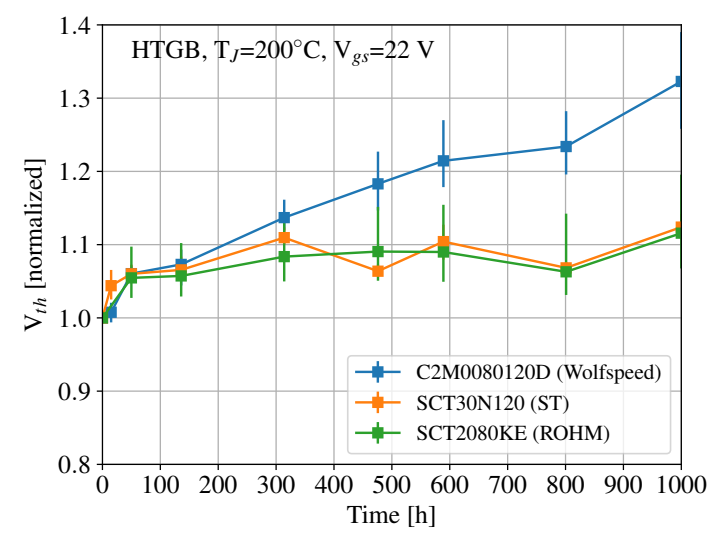

Figure 10: Evolution of the $V_{t h}$ for MOSFETs from three different manufacturers, in HTGB conditions $\left(V_{g s}=22 \mathrm{~V}\right)$, for an ambient temperature of $200^{\circ} \mathrm{C}$.

however, no obvious geometric parameter which could be correlated with the observed shift in $V_{t h}$.

\section{Discussion and Conclusion}

Recent SiC MOSFETs were tested, initially to evaluate the extend of know degradation mechanisms in static tests and realistic (switching) conditions: the drift in $V_{t h}$ caused by the voltage bias of the gate, and the increase in $V_{F}$ caused by the propagation of stacking faults in the crystal.

While none of these degradation mechanisms was found to have significant effects in our test conditions (this shows the progress of the SiC MOSFET technology in recent years), we observed a new degradation mechanism: the use of the body diode resulted in a strong drop in $V_{t h}$. In summary, the following conclusions can be drawn from the measurements presented above:

- for the Wolfspeed MOSFETs, static (Q3) and dynamic (CMB) tests have the same consequence, a large $V_{t h}$ drop occurring when current flows through the body diode, with comparable time constants in the drop;
- this drop depends on $V_{g s}$, with a larger change in $V_{t h}$ with $V_{g s}=-8 \mathrm{~V}(>40 \%$ after $80 \mathrm{~h})$ than with $V_{g s}=-5 \mathrm{~V}$ $(20 \%)$;

- in the absence of current biasing of the body diode (i.e HTRB/HTGB conditions, with only voltage bias, or Q1/Q4 conditions, with current biasing of the channel), no noticeable $V_{t h}$ drift is observed at $T_{J}=150^{\circ} \mathrm{C}$;

- increasing $T_{J}$ up to $200^{\circ} \mathrm{C}$ yields a noticeable $V_{t h}$ increase in HTGB conditions, but it remains much slower than that observed for Q3 or CMB tests (hundreds of hours vs. tens of hours)

- devices from different manufacturers (here ST Microelectronics and Rohm Semiconductor) appear to be much less sensitive to this $V_{t h}$ drift, which indicates that the issue can be mitigated through improvements in the device design or manufacturing processes;

- the $V_{t h}$ drift in $\mathrm{Q} 3$ or $\mathrm{CMB}$ conditions is correlated with a larger drift in HTGB conditions at $200^{\circ} \mathrm{C}$ (i.e. it affects Wolfspeed's MOSFETs much more than Rohm's or ST's), but the physical cause remains unknown. Cross sections of the three devices do not show fundamental differences in the geometry of these transistors.

The root cause of this mechanism is not fully understood yet: as depicted in Fig. 1, body diode current and channel current flow through different paths. Charge injection in the channel (which would cause a drift in $V_{t h}$ ) is therefore unlikely when using the body diode. For the same reason, it is not expected that the channel area would reach a higher temperature when using the body diode, as self heating occurs in a slightly different location. The power dissipation (and therefore the junction temperature) is identical for Q1, Q3 and Q4 tests, so a possible error in the estimation of the junction temperature would affect Q1, Q3 and Q4 test results identically, and yet the $V_{t h}$ drift is very different (see Fig. 7). This degradation is relatively severe, as it may make some devices impossible to turn off, or make them very sensitive to voltage transients on the gate within a few tens of hours of operation.

Qualifications test standards, such as HTRB or HTGB, were developed mainly for Si devices. With the development of $\mathrm{SiC}$ MOSFETs, some additional tests should be introduced. Here, we describe a very simple static test circuit (the Q3 test setup) which gives results which are consistent with those obtained in switching conditions. As shown in the paper, this test setup is capable of exposing weaknesses which were not visible after HTGB or HTRB tests, and yet may eventually cause some transistors to fail.

\section{Acknowledgement}

This work was funded as part of the GENOME-PREMICES initiative, in the scope of the "Programme d'Investissements d'Avenir" (Investment for the future) of CORAC (French Research Council for Civil Aeronautics). 


\section{References}

[1] K. Boomer, J.-M. Lauenstein, A. Hammoud, Body of knowledge for silicon carbide power electronics, Tech. rep., NASA (2016).

URL https://ntrs.nasa.gov/archive/nasa/casi.ntrs.nasa. gov/20170003922.pdf

[2] J. Millán, P. Godignon, X. Perpiñà, A. Pérez-Tomás, J. Rebollo, A Survey of Wide Bandgap Power Semiconductor Devices, IEEE transactions on Power Electronics 29 (5) (2014) 2155-2163.

[3] C. Raynaud, D. Tournier, H. Morel, D. Planson, Comparison of high voltage and high temperature performances of wide bandgap semiconductors for vertical power devices, Diamond and Related Materials 19 (1) (2010) 1-6. doi:DOI: 10.1016/j.diamond.2009.09.015.

[4] P. G. Neudeck, W. Huang, M. Dudley, Study of bulk and elementary screw dislocation assisted reverse breakdown in low-voltage $(<250 \mathrm{~V}) 4 \mathrm{H}-\mathrm{SiC}$ $\mathrm{p}+-\mathrm{n}$ junction diodes. i. dc properties, IEEE Transactions on Electron Devices 46 (3) (1999) 478-484. doi:10.1109/16.748865.

[5] T. Kimoto, N. Miyamoto, H. Matsunami, Performance limiting surface defects in SiC epitaxial p-n junction diodes, IEEE Transactions on Electron Devices 46 (3) (1999) 471-477. doi:10.1109/16.748864.

[6] R. Singh, Reliability and performance limitations in $\mathrm{SiC}$ power devices, Microelectronics and Reliability 46 (5-6) (2006) 713-730. doi:DOI: 10.1016/j.microrel.2005.10.013.

[7] P. G. Muzykov, R. M. Kennedy, Q. J. Zhang, C. Capell, A. Burk, A. Agarwal, T. S. Sudarshan, Physical phenomena affecting performance and reliability of $4 \mathrm{H}-\mathrm{SiC}$ bipolar junction transistors, Microelectronics Reliability In Press, Corrected Proof (2008) -. doi:DOI: 10.1016/j.microrel.2008.10.009.

[8] H. Chen, B. Raghothamachar, W. Vetter, M. Dudley, Y. Wang, B. J. Skromme, Effects of different defect types on the performance of devices fabricated on a $4 \mathrm{H}-\mathrm{SiC}$ homoepitaxial layer, MRS Proceedings 911. doi:10.1557/PROC-0911-B12-03.

[9] A. J. Lelis, R. Green, D. B. Habersat, M. El, Basic Mechanisms of Threshold-Voltage Instability and Implications for Reliability Testing of SiC MOSFETs, IEEE Transactions on Electron Devices 62 (2) (2015) 316-323. doi:10.1109/TED.2014.2356172.

[10] L. Yang, A. Castellazzi, High temperature gate-bias and reverse-bias tests on $\mathrm{SiC}$ MOSFETs, Microelectronics Reliability 53 (9) (2013) 1771 - 1773, european Symposium on Reliability of Electron Devices, Failure Physics and Analysis. doi:https://doi.org/10.1016/j.microrel.2013.07.065.

[11] O. Kusumoto, A. Ohoka, N. Horikawa, K. Tanaka, M. Niwayama, M. Uchida, Y. Kanzawa, K. Sawada, T. Ueda, Reliability of DiodeIntegrated SiC Power MOSFET (DioMOS), Microelectronics Reliability 58 (2016) 158 - 163, reliability Issues in Power Electronics. doi:https://doi.org/10.1016/j.microrel.2015.11.033.

[12] A. Fayyaz, G. Romano, A. Castellazzi, Body diode reliability investigation of sic power mosfets, Microelectronics Reliability 64 (2016) 530 - 534, proceedings of the 27th European Symposium on Reliability of Electron Devices, Failure Physics and Analysis. doi:https://doi.org/10.1016/j.microrel.2016.07.044.

[13] JESD22-A108D, temperature, bias and operating life (2010). URL www. jedec. org

[14] Wolfspeed, C2M0080120D Silicon Carbide Power MOSFET, C2M MOSFET Technology (2018).

URL https : //www. wolfspeed.com/

[15] B. H. Nya, J. Brombach, D. Schulz, Benefits of higher voltage levels in aircraft electrical power systems, in: 2012 Electrical Systems for Aircraft, Railway and Ship Propulsion, 2012, pp. 1-5. doi:10.1109/ESARS.2012.6387381.

[16] Y. Avenas, L. Dupont, Z. Khatir, Temperature measurement of power semiconductor devices by thermo-sensitive electrical parameters - a review, IEEE Transactions on Power Electronics 27 (6) (2012) 3081-3092. doi:10.1109/TPEL.2011.2178433.

[17] S. Microelectronics, SCT30N120 Datasheet (2017). URL http: //www. st. com/

[18] R. Semiconductor, Sct2080ke datasheet (2017).

URL http: //www . rohm. com/ 